\title{
Contributions for Ethernet Based Networking in Manufacturing Systems
}

\author{
Sérgio E. Loureiro, Adriano S. Carvalho \\ Faculdade de Engenharia da Universidade do Porto \\ Instituto de Sistemas e Robótica - Grupo de Automação Industrial \\ R. dos Bragas 4099 Porto Codex - Portugal \\ Tel: 02 -2041619 email sel@tormentas.fe.up.pt, asc@garfield.fe.up.pt
}

\begin{abstract}
The work presented is this communication intends to evaluate the possibility of using Ethernet to perform integration in manufacturing systems. The principal obstacle is its random medium access time that is not suitable to real-time systems. It refers to some experimental tests based on a case study, which showed that Ethernet can be a correct choice in soft real-time systems. Furthermore, it is discussed the design and implementation issues behind the alteration of the medium access control of Ethemet to support real-time, without requiring any modifications of hardware.
\end{abstract}

Keywords
communication networks, ethernet, real-time communication, CNMA

\section{INTRODUCTION}

The integration between the different levels of a manufacturing system is a cumbersome task. This results from the diversity of equipments and different communication requirements among the several manufacturing levels.

Ethernet could be a useful alternative, due to its availability in most equipments and low price. However, this option has some associate problems, like the possible unlimited time response. This fact does not allow Ethernet utilisation at the lower levels of a manufacturing system, where hard real-time is usually required.

Nevertheless, in soft real-time systems, we can evaluate an ethernet based solution, realising that its medium access time is (probably) almost zero for light loads. This great advantage in comparison to another LANs has become more attractive with the availability of higher bandwidth Ethernets. On the other hand, this fact is not guaranteed, so the application must dynamically cope with possible late messages.

It is not possible to establish rules between load and access time, since there are factors that influence Ethernet medium access time, like traffic pattern and packet size (Molle, 1994).

So, if either this probabilistic approach does not cope with application requirements, or it is a hard real-time system, then Ethernet utilisation is not possible. The alternatives are fieldbuses, 
token-bus or Ethernet adapted to real-time. This last possibility must be evaluated in terms of cost, implementation and integration effectiveness.

\section{SOFT REAL-TIME SYSTEMS}

This section tries to clarify ethernet utilisation in soft real-time systems and to identify the associate problems.

Firstly, the medium access control is contention-based, that is, there is not any access restriction but the channel is idle. This implies the possibility of occurring collisions, that are resolved in a nondeterministic way. However, we must say that the time involved in resolving one collision is not the problem, because it is very low. The real problem appears for successive collisions with the same packet, which is unlikely to happen under light load.

Secondly, the service provided by Ethernet protocol at different stations is not fair (Boggs, 1988), and its performance is dependent on packet size.

Thirdly, there is the capture effect. This effect results from hardware evolution and affects the successful transmission probability of the packets that are involved in successive collisions, improving the conditions for their starvation (the retransmissions are aborted after 16 tries). This effect can happen at low utilisation, of about $45 \%$.

Some experimental tests were conducted to clarify these points. These problems, namely the capture effect were reported in high performance platforms (workstations) and with traffic very distinct from the one found in manufacturing systems The main goal is to verify if the referred obstacles to Ethernet utilisation happened in lower performance platforms (PCs in our case), that are usually present in these systems and to try to evaluate their consequences. The test conditions were based on a case study described on (Loureiro, 1996) and (Praça, 1996) that concerns an integrated application constituted by planning, scheduling, simulation and monitoring modules developed to a textile industry.

A network constituted by three i 486 based PCs running Linux v2.0.6 was used, with off-the-shelf Ethernet cards (SMC-Ultra and D-Link).

Firstly, we try to characterise the test platform. The results showed that just one PC can absorb the entire bandwidth, but only if it is dedicated to this job and with large dimension packets $(1,5$ Kbytes). With small dimension packets (64 bytes) the results found are very different; the maximum bandwidth occupied becomes about $15 \%$ with one PC.

This fact constituted a limitation of the tests because we cannot evaluate high utilisations with low dimension packets. In spite of using a workstation with the objective of absorbing bandwidth, we continued not to achieve high utilisations with small dimension packets.

After these characterisation platform tests, we have tested the Ethernet service time. We define service time as being the time necessary to transmit a request and receive the related acknowledgement. The sizes of packets tested were the small dimension ones because it is usually sufficient to transmit process variables, alarms, control commands and acknowledgements, and 1 Kbytes because it is the maximum size allowed by standard manufacturing protocols. The transfer of large pieces of data, for example the download of programs, is properly performed with large packets.

Nevertheless, we have registered packets dropped due to excessive collisions (and some packets with large service times), when about half of the entire bandwidth was occupied with large packets and we tried to transmit a few small dimension packets and related acknowledgements.

In cases with greater bandwidth occupied (inferior at $80 \%$ ), but with identical dimension packets, we have not registered any dropped packets. This result reaffirms a guideline of (Boggs, 1988) that 
advises not to mix very different dimension packets in the same network segment if we want to achieve high performances.

In spite of the tests' limitations, namely the number of hosts, we believe that Ethernet utilisation can be a correct solution for soft real-time systems.

The main constraint is that it is not possible to limit network utilisation in a given moment, or to make a bandwidth reservation to a host. We have to pay particular attention to possible time slots occupied with large dimension packets.

\section{HARD REAL-TIME SYSTEMS}

If the application context is of hard real-time systems, we cannot use Ethernet. Nevertheless, we can make use of low cost Ethernet cards to develop a solution that supports real-time traffic.

The option of altering the Ethernet medium access control without requiring any modifications of existing hardware was made, not only for reasons of low cost, but also to allow a more generic and flexible implementation. The aim of this section is to discuss the design and implementation of a software based proposal.

After an analysis of the off-the-shelf Ethernet cards and network interface controllers, we realise that it is impossible to change the medium access method and the retransmission algorithm, since they have been implemented in firmware.

This fact does not allow to change the medium access to a method that resolves collisions in a deterministic way like CSMA/DCR or CSMA/PDCR (Turiel, 1996). When a packet is copied to the controller transmission buffer, we have no more control over it.

There are some network interface controllers that constitute an exception to the last two affirmations because they allow to disable retransmissions after the first collision of a packet. The use of any these proposals would restrict the final solution applicability.

What leave us the possibility of using methods that guarantee an exclusive medium access. These methods can always work in an exclusive manner or with a way of changing between two modes of operation: a controlled one and a normal one. The work presented in (Venkatramani, 1995) describes one protocol of the second type. However, this kind of protocol brings up an additional problem that is the transition between modes of operation. (Venkatramani, 1995) proposes a mechanism of broadcasting one message to perform it.

Another possibility is to define an interval where each host has exclusive medium access and can request a change of operating mode. The definition of this interval can be made by clock synchronisation or by an established order.

This interval of exclusive medium access can also serve to make a reservation of a time slot, or to establish priorities among hosts like in (Adelstein, 1994).

In applications where there are periodic sources, we can use the information about the size and periodicity of their transmissions to assign exclusive time slots to these sources by means of a distributed algorithm like the one described in (Yavatkar, 1992).

So, we can conclude that there are several methods to implement, and the pros and cons of each proposal result from a trade-off between efficiency, implementation and access priorities.

A drawback of this software based solution is that it does not guarantee deadlines if integrated into standard ethernet network segments, that is not implementing our medium access modifications. 


\section{IMPLEMENTATION}

In the same tests platform, already described, we developed a generic software based solution that allows the implementation of any of these proposals.

There were developed two distinct modules: one that catches traffic information, and another that restricts the medium access.

The module that gets traffic information is done operating the network controller in promiscuous mode (present in the all controllers analysed). In this easy way, we can get all possible traffic information, that allows the design of other methods, namely predictive or closed loop methods. The only drawback of this solution is the extra processing time needed.

The module that restricts medium access, or better, card access is implemented at the device driver level, more specifically, in the device independent routines, which implies that our solution supports any network card, without requiring any modifications on the respective device driver. Also, it supports any protocol in spite of only having tested TCP/IP.

This module implied kernel modifications, so we created a channel between this kernel part and the user space, which allows an easier implementation of all the methods described.

This constitutes a generic platform, capable of performing the tests set discussed before and new tests involving other methods of control, in order to analyse solutions that can cope with the requirements of hard real-time systems.

\section{CONCLUSIONS}

The Ethernet protocol is an useful alternative to achieve manufacturing systems integration. In real time operating conditions, we must evaluate the application requirements and decide in conformance. If the communication requirements are not copped by this solution, it is necessary to implement appropriate medium access control. It is our intention to develop a method library that suits the most common application.

\section{REFERENCES}

Boggs, David R., Mogul, Jeffrey C. and Kent, Christopher A. (1988) Measured Capacity of an Ethernet: Myths and Reality. Proceedings of the ACM SIGCOMM 88.

Molle, Mart L. (1994) A New Binary Logarithmic Arbitration Method for Ethernet. University of Toronto.

Praça, Isabel C. (1996) Balanceamento e Simulação de Linhas de Fabrico Manuais. Faculdade de Engenharia da Universidade do Porto.

Loureiro, Sérgio E. (1996) A rede Ethernet como elemento integrador dos sistemas de fabrico. Faculdade de Engenharia da Universidade do Porto.

Turiel, Javier Pérez, Marinero, Juan C. Fraile and González, Jose R. Perán (1996) CSMA/PDCR: A Random Access Protocol Without Priority Inversion. IEEE Proceedings of the IECON.

Venkatramani, Chitra and Chiueh, Tzi-cker (1995) Design, Implementation, and Evaluation of an Software-based Real-Time Ethernet Protocol. Proceedings of the ACM SIGCOMM.

Yavatkar, Rajendra, Pai, Prashant and Finkel, Raphael (1992) A Reservation-based CSMA Protocol for Integrated Manufacturing Networks. University of Kentucky. 